\title{
Orthogonal Functionalization of Nanoporous Substrates: Control of 3D Surface Functionality
}

\author{
Thomas D. Lazzara, ${ }^{\dagger}$ Torben-Tobias Kliesch, ${ }^{\dagger}$ Andreas Janshoff, ${ }^{\ddagger}$ and Claudia Steinem ${ }^{*,+}$ \\ ${ }^{\dagger}$ Institute of Organic and Biomolecular Chemistry, Tammannstrasse 2, 37077 Göttingen, Germany \\ ${ }^{\ddagger}$ Institute of Physical Chemistry, Tammannstrasse 6, 37077 Göttingen, Germany \\ Supporting Information
}

\begin{abstract}
Anodic aluminum oxide (AAO) membranes with aligned, cylindrical, nonintersecting pores were selectively functionalized in order to create dual-functionality substrates with different pore-rim and pore-interior surface functionalities, using silane chemistry. We used a two-step process involving an evaporated thin gold film to protect the underlying surface functionality of the pore rims. Subsequent treatment with oxygen plasma of the modified AAO membrane removed the unprotected organic functional groups, i.e., the pore-interior surface. After gold removal, the substrate became optically transparent, and displayed two distinct surface functionalities,

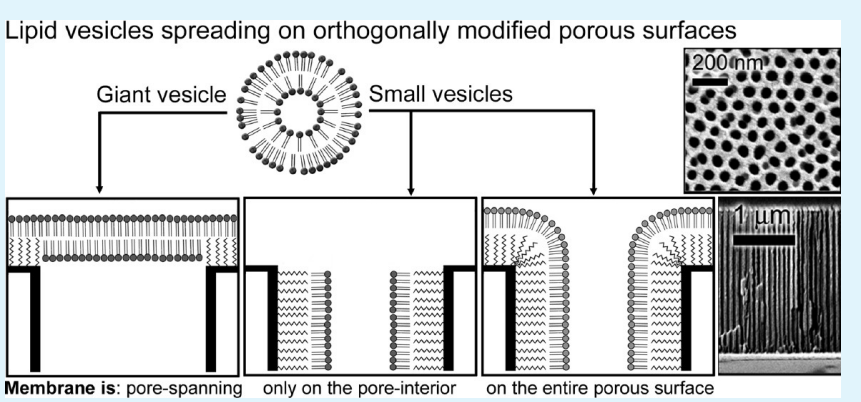
one at the pore-rim surface and another at the pore-interior surface. We achieved a selective hydrophobic functionalization with dodecyl-trichlorosilane of either the pore rims or the pore interiors. The deposition of planar lipid membranes on the functionalized areas by addition of small unilamellar vesicles occurred in a predetermined fashion. Small unilamellar vesicles only ruptured upon contact with the hydrophobic substrate regions forming solid supported hybrid bilayers. In addition, pore-rim functionalization with dodecyl-trichlorosilane allowed the formation of pore-spanning hybrid lipid membranes as a result of giant unilamellar vesicle rupture. Confocal laser scanning microscopy was employed to identify the selective spatial localization of the adsorbed fluorescently labeled lipids. The corresponding increase in the AAO refractive index due to lipid adsorption on the hydrophobic regions was monitored by optical waveguide spectroscopy. This simple orthogonal functionalization route is a promising method to control the three-dimensional surface functionality of nanoporous films.
\end{abstract}

KEYWORDS: nanoporous substrates, optical light mode waveguide spectroscopy, orthogonal silanization, pore-spanning lipid membranes, reactive plasma, silane chemistry

\section{INTRODUCTION}

Anodic aluminum oxide (AAO) is one of the most promising ordered nanoporous materials. It has been widely used for its selforganized predictable structure that is composed of nonintersecting, hexagonally close-packed, cylindrical pores with conveniently adjustable monodisperse pore diameters, degree of lattice spacing, and membrane thickness. ${ }^{1-4}$ AAO membranes have $1-3$ orders of magnitude increased surface area because of their high porosity; for example, a $1 \mathrm{~cm}^{2}$ AAO substrate with an interpore spacing of $100 \mathrm{~nm}$, a thickness of $4 \mu \mathrm{m}$ and pore diameters of $60 \mathrm{~nm}$, has $1.2 \times 10^{10}$ pores $\mathrm{cm}^{-1}$ and a total surface area of $90 \mathrm{~cm}^{2}$.

A range of chemical strategies is available to chemically modify planar substrates. Coinage metals, ${ }^{5-7}$ polymers, ${ }^{8,9}$ and inorganic or metal oxide ${ }^{10-12}$ substrates can be functionalized by the formation of functional monolayers through surface reactions such as silanizations, ${ }^{13}$ reactive plasma treatments, ${ }^{14,15}$ thiol and phosphonate chemistry. ${ }^{16}$ Although a homogeneous deposition of pores molecules on such surfaces is rather straightforward, tailored micropatterned chemistry is a more demanding task, but essential for designing locally addressable areas on substrates. In general, the areas to be addressed need to be selectively treated or protected, which requires an orthogonal functionalization procedure. At the micrometer scale, template-based methods are commonly used to selectively evaporate metals, or to cure photoresist films. Soft-lithography ${ }^{17}$ is applied to create micrometer-sized and spatially localized surfaces. At the submicrometer scale, more elaborate template procedures have been developed for orthogonal functionalization such as using nanoporous alumina films as a patterning mask to create ordered metal nanodot arrays ${ }^{18}$ or to localize arrays of seed catalytic sites. $^{19}$

While selective functionalization methodologies have been developed for flat surfaces, ${ }^{20-22}$ porous 3-dimensional substrates still remain a challenge for orthogonal functionalization, which can produce selective, spatially distinct surface chemistries. The orthogonal functionalization of porous materials would be an important advancement in technological fields such as separation and sieving applications, as well as high-sensitivity bifunctional

Received: December 9, 2010

Accepted: $\quad$ March 3, 2011

Published: March 03, 2011 
detection platforms. AAO has already proven useful in nondestructive, high sensitivity assays such the selective separation of drug enantiomers ${ }^{23,24}$ or DNA oligomers, ${ }^{25}$ the development of on-chip biosensors, ${ }^{26-28}$ for tailoring molecular transport properties, ${ }^{29,30}$ and more recently for energy storage devices. ${ }^{31}$

We have shown in the past that orthogonal functionalization of porous substrates can be achieved by directly depositing a gold metal layer on the substrate, which then allows pore-rim modification using functional thiols designed to direct the formation of lipid membranes. ${ }^{32,33}$ However, for nanoporous materials with pore diameters below the scattering limit $(<100 \mathrm{~nm})$, the attractive advantage of optical transparency is lost when a metal is deposited onto the porous film. Hence, this strategy prevents the study of processes occurring within the porous network using various optical methods. Silane-based orthogonal functionalizations of AAO appear to be well-suited to circumvent this problem, ${ }^{34-36}$ but challenging to implement because spatial selectivity is difficult to achieve. Voelcker and co-workers ${ }^{37}$ report on a silane-based strategy to selectively functionalize the aluminum surface before the anodization process that generates $\mathrm{AAO}$, which results in a selective functionalization of the pore rims. In this procedure, the deposited molecules must endure the anodization process, as well as pore widening in acidic solution. Thus, it is not suited for functional moieties that are moisture- or $\mathrm{pH}$-sensitive. Other orthogonal functionalization strategies have been reported for nanoporous substrates, but are technically more demanding and offer lower control on both, the degree of functionalization and spatial localization of the functionality. ${ }^{38}$ Kilian et al. ${ }^{39}$ used surface tension and capillary forces to facilitate or prevent pore-interior functionalization. Sailor and coworkers ${ }^{40}$ produced porous silicon that was etched in a two-step process involving a hydrophobic functionalization after the first step to produce a membrane with dual-functionality. A porous membrane with hydrophilic pore rims and hydrophobic pore interiors was achieved using plasma-polymerization of a fluorocarbon layer. $^{41}$ Recently, the wetting properties of AAO were tuned by the selective deposition, by electrospray technique, of a thin polymer film at the pore rims. ${ }^{42}$

In this contribution, we present a simple technique, where $\mathrm{AAO}$ silanization is performed after the anodization and pore-widening processes, i.e., on the desired final substrate, which ensures that the surface functionality is created just before use, therefore ensuring optimal chemical integrity, degree of functionalization and spatial localization of the surface chemistry. The presented method is based on a thin evaporated gold layer serving as a protective mask that prevents the removal of the functionalization from the underlying surface by the reactive oxygen plasma, which is used to remove the undesired surface functionality on the unprotected surface. We have used a hydrophobic silane, namely dodecyl-trichlorosilane, to differentially functionalize the AAO substrates and demonstrated that fluorescently labeled phospholipid vesicles interact only with the hydrophobic surface, i.e., pore-rim or pore-interior surface, or both. Depending on the size of the phospholipid vesicles, we were able to produce hybrid solid-supported lipid membranes on the pore-interior AAO surface using small unilamellar vesicles (SUVs), or hybrid porespanning membranes on functionalized AAO with hydrophobic pore-rim surfaces using giant unilamellar vesicles (GUVs).

\section{RESULTS AND DISCUSSION}

The preparation of selectively functionalized nanoporous substrates using silane chemistry is challenging because the

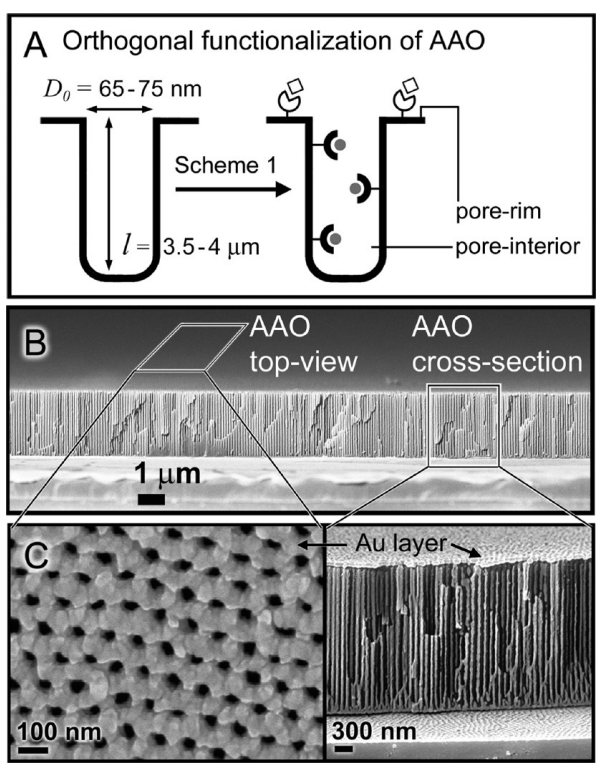

Figure 1. (A) AAO membranes with $D_{0}$ not larger than the scattering limit $\left(\sim 1 / 10 \lambda_{\text {light }}\right)$ can be used in applications requiring dual-functionality and optical transparency. (B) Scanning electron microscopy image of an AAO membrane, which was used as a thin-film mounted on a glass substrate using an optical adhesive. (C) Enlarged top and cross-section views of $\mathrm{B}$. Au was evaporated onto the $\mathrm{AAO}$ as a protective mask for the pore rims; a rather thick Au layer (about $50 \mathrm{~nm}$ ) was evaporated to emphasize its location atop the AAO pores, as shown in the top view (left) and the cross-section view (right). A $10-20 \mathrm{~nm} \mathrm{Au}$ layer is sufficient to create the protective layer for the orthogonal functionalization.

porous surface reacts homogeneously and typical planar surface patterning techniques are generally not applicable to porous structures. Therefore, the development of new functionalization strategies that can discriminate between the pore-rim and poreinterior surfaces are required. For our studies, we used anodic aluminum oxide (AAO), which was produced by anodizing aluminum in $0.3 \mathrm{M}$ oxalic acid under a constant potential of $40 \mathrm{~V}$. This procedure resulted in AAO substrates with an interpore distance of $p=100 \mathrm{~nm}$, pore diameters that were enlarged to $D_{0}=$ $65-75 \mathrm{~nm}$ and with a chosen thickness of $l=3.5-4.0 \mu \mathrm{m}$ (Figure 1A).

The AAO membranes were covered with a thin metal coupling layer ( $2 \mathrm{~nm}$ of $\mathrm{Cr}$ and $25 \mathrm{~nm}$ of $\mathrm{Au}$ ) on the aluminum oxide barrier side (bottom) and then mounted on glass supports using an optical adhesive ${ }^{43}$ (Figure $1 \mathrm{~B}$ ) to allow the characterization of their refractive index using optical waveguide spectroscopy (OWS). The AAO pores were hexagonally ordered (Figure 1C, left image) with a cylindrical geometry (Figure 1C, right image). On the AAO substrate shown in Figure $1 \mathrm{C}$, an excess of approximately $50 \mathrm{~nm}$ Au was evaporated on the top to emphasize the location of the protective metal masking layer that is used to generate the orthogonal functionalization, i.e., the localized pore-rim surface functionalization.

Functionalization Strategies. Two different routes were developed to orthogonally functionalize the AAO substrates (Scheme 1 , steps $1-5$ and $A-D$ ). In both strategies, a 10$20 \mathrm{~nm}$ thick Au film serves as a protection layer preventing the underlying pore-rim surface to be chemically modified in further steps. We rely on the significantly larger pore-interior surface area, in comparison to the pore-rim surface area, to minimize the 
Scheme 1. Two experimental protocols $(1-5$ and A-D) to prepare AAO substrates with dual-functionality using a thin Au layer as a protective mask (gray box). Thermally evaporated gold is used to prevent the underlying AAO pore-rim functionalization from oxidation during $\mathrm{O}_{2}$ plasma treatment. The final AAO substrates, after step 5 or step $\mathrm{D}$, have different pore-rim vs pore-interior functionalities and most importantly, remain optically transparent

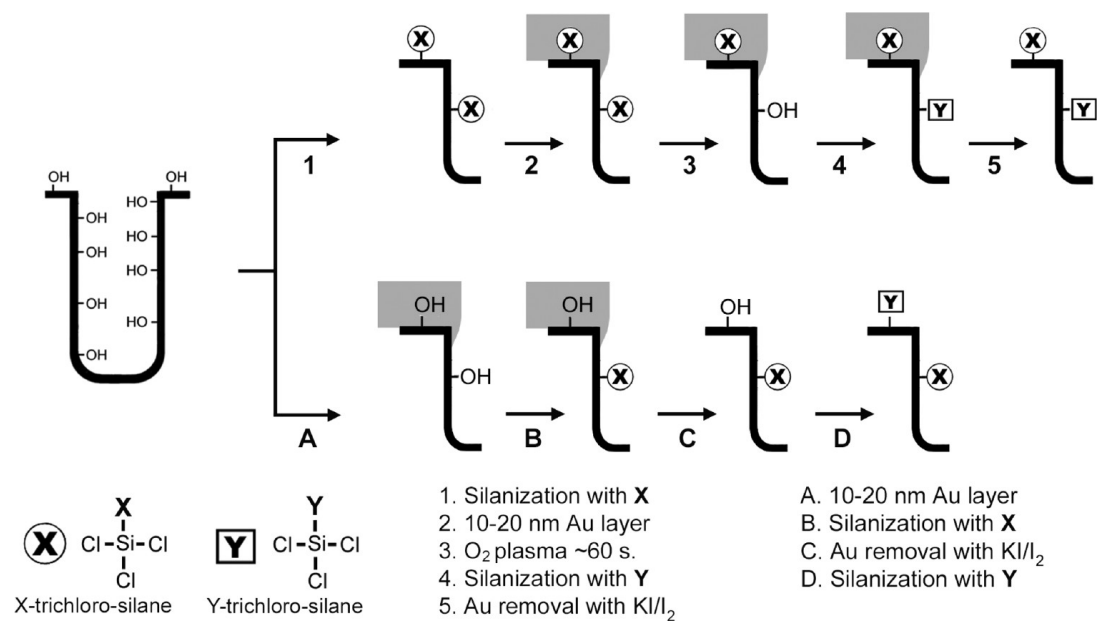

degree of pore-interior contamination with $\mathrm{Au}$. Under high evaporation rate conditions $\left(1-2 \mathrm{~nm} \mathrm{~s}^{-1}\right)$ we did not observe significant Au deposition within the cavities of the substrate. Even for $50 \mathrm{~nm}$ of evaporated $\mathrm{Au}$, we observed that the metal only forms small clusters within the AAO pores (see the Supporting Information, Figure S4). The importance of the interior contamination is reduced by the large aspect-ratio of the AAO, and the degree of contamination can be estimated by the ratio of the pore area to pore-interior surface, which is $<2 \%$ for a $4 \mu \mathrm{m}$ thick AAO film.

Following route $1-5$ (Scheme 1), the hydroxyl terminated $\mathrm{Al}_{2} \mathrm{O}_{3}$ surface is first silanized to obtain the desired surface chemistry $(-\mathrm{X})$ on the aluminum oxide (step 1$)$. Second, a protective Au layer is evaporated (step 2) covering the pore rims, followed by an $\mathrm{O}_{2}$ plasma treatment for about $60 \mathrm{~s}$ (step 3). During $\mathrm{O}_{2}$ plasma treatment, organic groups are removed from the surface mainly via reaction with reactive radical species generated in the ionized gas. ${ }^{44-46}$ The AAO surface functionalized with the organic silane present beneath the Au film remains intact, while the organic functionality within the pore interior is oxidized and removed. The hydroxyl terminated surface generated on the pore interiors is thereby ready for a second silanization (-Y) (step 4). Finally, (step 5) the Au film is removed using an iodine $\left(\mathrm{KI} / \mathrm{I}_{2}\right)$ solution to obtain a metal-free orthogonally functionalized AAO substrate. The chemical integrity of the remaining functional groups was proven by fluorescence microscopy (see Supporting Information, Figure S1). The second strategy starts by evaporating a protective Au layer onto the AAO substrate (step A), followed by silanization $(-X)$ of the remaining accessible $\mathrm{Al}_{2} \mathrm{O}_{3}$ pore-interior surface (step B). Removal of the gold layer by an iodine solution $\left(\mathrm{KI} / \mathrm{I}_{2}\right)$ (step $\mathrm{C}$ ) generates a hydroxyl terminated surface on the pore rims, which can be functionalized with a second silanization reaction $(-Y)$ (step D). We have employed both strategies to deposit lipid membranes by vesicle rupture on porous alumina surfaces, which were functionalized with dodecyl-trichlorosilane $\left(\mathrm{C}_{12}\right.$-TCS $)$ on different areas.

Selective Deposition of Hybrid Solid Supported Lipid Membranes. According to the two procedures depicted in Scheme 1 , we prepared AAO membranes with a hydrophobic
$\mathrm{C}_{12}$-TCS layer at different positions (Figure 2A). Substrate 1 was functionalized entirely with $\mathrm{C}_{12}$-TCS such that pore rims and pore interiors were both hydrophobic. Substrate $\mathbf{2}$ was prepared according to steps $\mathrm{A}-\mathrm{D}$, without performing the last step (Scheme 1), resulting in pore rims that are hydrophilic and pore interiors that are hydrophobic after silanization with $\mathrm{C}_{12}$-TCS. Substrate 3 is the inverse of 2 , with hydrophobic pore rims, prepared by following steps $1-5$ (Scheme 1). Substrate 4 was subjected to $\mathrm{O}_{2}$ plasma treatment rendering it hydrophilic and nonfunctionalized and, as such, used as reference (Figure 2A).

The substrates were incubated with fluorescently labeled small unilamellar vesicles (SUVs) composed of 1-palmitoyl-2-oleoylsn-glycero-3-phosphocholine (POPC). The weighted-number SUV size distribution, measured by dynamic light scattering, ranged between $20-30 \mathrm{~nm}$ in diameter. SUVs are known to adhere strongly on different hydrophobic functionalities and can rupture to form hybrid solid-supported lipid monolayers ${ }^{47}$ on alkyl-terminated hydrophobic surfaces. It has also been shown that SUVs do not adsorb on native hydrophilic $\mathrm{Al}_{2} \mathrm{O}_{3}$ surfaces. ${ }^{47}$ Confocal laser scanning microscopy (CLSM) images in $Y-Z$ plane of substrates $\mathbf{1}-\mathbf{4}$ were taken to localize the lipids (Figure 2B, left-side). Substrate $\mathbf{1}$ and $\mathbf{2}$ show a strong fluorescence throughout the $Y-Z$ planes indicating that lipids are lining the pore interiors throughout the AAO. For substrate 3, fluorescence is not observed in the pore interior but only atop due to surface adsorbed vesicles. The bottom of the AAO substrate can be readily located by a reflection owing to the metal coupling layer on the AAO barrier side. For CLSM scans performed in the Z-direction, the resolution is around $0.9 \mu \mathrm{m}$ for a numerical aperture of 1 . For images in the $Y-Z$ plane, multiple thin slides of about $100 \mathrm{~nm}$ thick were taken, over the $10-15 \mu \mathrm{m}$ vertical range that was scanned, in order to improve the spatial localization of the fluorescence.

Substrate $\mathbf{4}$ does not show any significant fluorescence, only a weak autofluorescence, as the SUVs adsorb weakly on the nonfunctionalized AAO. Epifluorescence images of substrates 1 and 3 (Figure 2B, right-side) taken under identical experimental and exposure conditions show that hybrid solid supported lipid membranes formed within the AAO pore interiors (substrate 1), which 

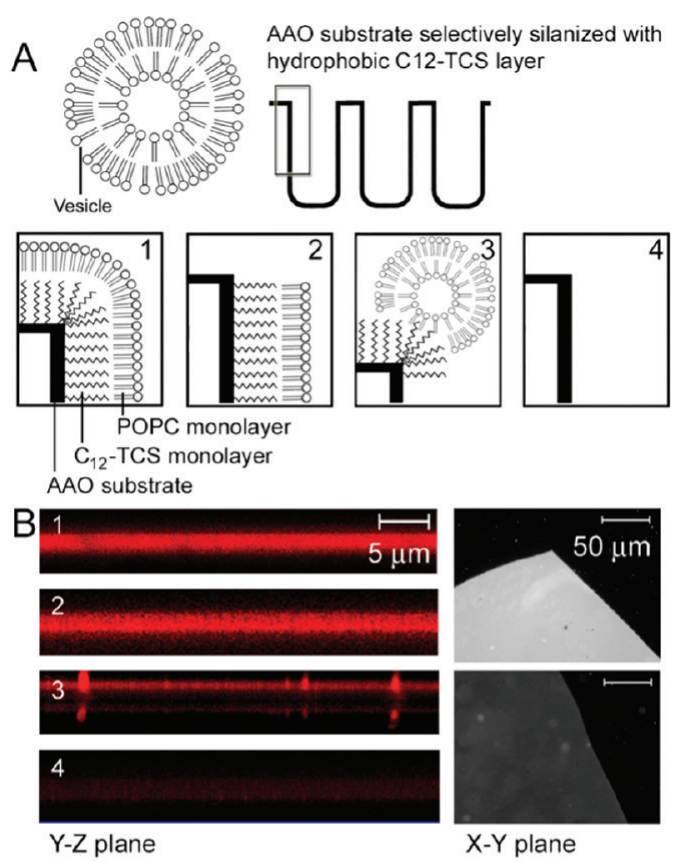

C

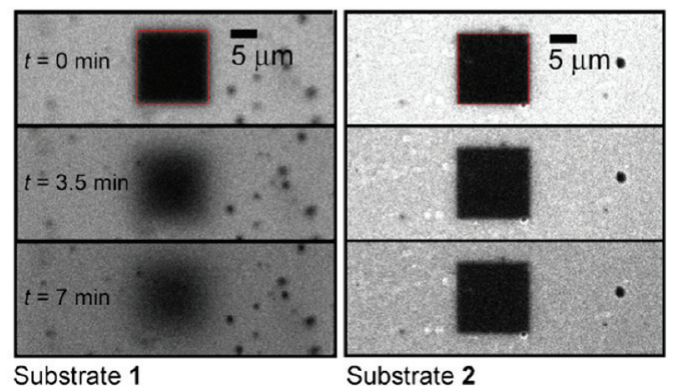

Figure 2. (A) Schematic representation of AAO substrates differing in their orthogonal surface functionalities. Small unilamellar POPC vesicles are expected to interact differently with these surfaces. (B) Fluorescence images of the substrates with $D_{0}=75 \mathrm{~nm}$ and $l=3.8 \mu \mathrm{m}$ after addition of SUVs are shown. The left side shows CLSM fluorescence cross-sectional $Y-Z$ plane images for AAO substrates $1-4$. The images on the right are top $X-Y$ plane epi-fluorescence images of substrates (top) 1 and 3 (bottom) under identical exposure conditions showing the increased fluorescence intensity of $\mathbf{1}$ due to lipids adsorbed on the entire AAO surface vs only atop for 3. (C) FRAP experiments on substrate $\mathbf{1}$ and 2, to which SUVs doped with Bodipy-DHPE were added.

increases significantly the overall fluorescence intensity compared to only surface adsorbed lipids (substrate 3 ). The epifluorescence images were taken at the AAO substrate edge, where the optical glue is also visible to emphasize the contrast. The left-hand side of Figure $2 \mathrm{~B}$ shows confocal scans in the Z-direction that were taken for all 4 substrates with similar exposure conditions. To analyze whether a hybrid solid supported continuous lipid monolayer has been formed on substrate $\mathbf{1}$, which is not expected for substrate 2, fluorescence recovery after photobleaching (FRAP) experiments were performed on both these substrates using POPC SUVs doped with $0.1 \mathrm{~mol} \%$ Bodipy-DHPE. The fluorescence of a square region was photobleached (Figure 2C) and its recovery monitored. Significant fluorescence recovery was found for substrate $\mathbf{1}$. This observation is consistent with the scenario sketched in Figure 2A for substrate 1. A continuous lipid monolayer on top of the hydrophobic $\mathrm{C}_{12}$-TCS monolayer is

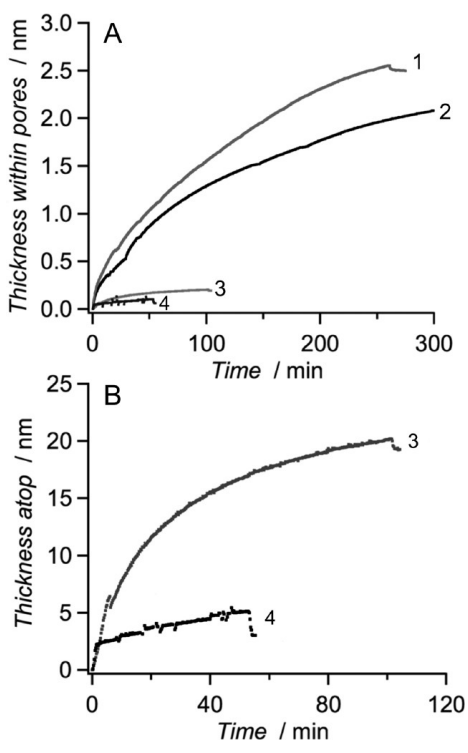

Figure 3. (A) Kinetics of POPC SUV interaction with the differently functionalized substrates $(\mathbf{1}-\mathbf{4})$ presented in Figure 2A measured using OWS. The overall dielectric constant of the AAO membrane with $D_{0}=$ $75 \mathrm{~nm}$ and $l=3.8 \mu \mathrm{m}$ increases because of the deposition of a 2.0-2.5 nm phospholipid monolayer within the pores for substrates 1 and 2, which is not observed for substrates 3 and 4. (B) The atop deposition of vesicles is much larger for substrate 3 than for $\mathbf{4}$ because only the hydrophobic pore rims of substrate 3 interact strongly with SUVs.

formed, which results in laterally mobile lipids. In contrast, no recovery was observed for substrate 2 within the time scale of the experiment. In this case (Figure 2A, substrate 2 ), the pore rims are not covered with lipids but are composed of $\mathrm{Al}_{2} \mathrm{O}_{3}$ serving as a barrier for the lipids to laterally move and therefore, fluorescence recovery is not expected.

In addition to the fluorescence image analysis, the kinetics of interaction of the POPC SUVs with the substrates $1-4$ were analyzed by time-resolved OWS measurements (Figure 3). For constant thickness samples, OWS provides information on the overall change in the dielectric constant of the optically transparent films. A uniform increase in the AAO dielectric constant can be observed as significantly large and uniform positive angular shifts in all of the waveguide modes, if lipids adsorb within the pores (see the Supporting Information, Figure S2). If vesicle adsorption only takes place atop the AAO film, only the higher order modes slightly shift while the lower order modes remain unchanged. OWS measurements of substrates $\mathbf{1}$ and $\mathbf{2}$ demonstrate that a dielectric layer with a thickness of about $2.0-2.5 \mathrm{~nm}$ has been adsorbed on the either fully functionalized (1) or inner-pore wall (2) functionalized AAO substrate. This change in thickness supports the notion of the formation of a hybrid solid supported lipid bilayer obtained by spreading of the SUVs on the hydrophobic $\mathrm{C}_{12}$-TCS monolayer.

In the case of substrates 3 and 4 , no significant interior deposition was observed, indicated by the very small change in optical thickness. However, for substrate 3 a significant atop SUV deposition with a thickness change of about $25 \mathrm{~nm}$ was observed, which was considerably larger than that observed for substrate 4, which was only about $2 \mathrm{~nm}$. This result confirms that intact SUVs adhere only on the hydrophobic monolayer on the pore rims, while they did not bind to native $\mathrm{Al}_{2} \mathrm{O}_{3}$ surfaces. The vesicles do not spread because of the limited contact area with the substrate. 
The results clearly demonstrate that a selective functionalization of the AAO substrates is feasible. We next asked the question, whether the developed strategy allows us to pattern the substrates, i.e., to generate laterally distinct areas of the AAO substrates.

Patterned Substrates. As the deposition of gold is a key step in generating a selective functionalization of the AAO substrates, we used a TEM grid with rectangular openings as a patterning-mask for Au deposition to laterally define rectangular areas that were to remain functionalized after plasma treatment. AAO substrates with hydrophobic pore rims were again obtained by silanization with $\mathrm{C}_{12}$-TCS. Gold was subsequently evaporated through the TEM grid (see inset of Figure 4A) to form rectangular patterns on the surface and then $\mathrm{O}_{2}$ plasma treated, followed by Au removal leading to substrate 3 (Figure 2A). To confirm the presence of hydrophobic pore rims, sonicated POPC SUVs doped with TexasRed-DHPE were added.

In Figure 4, fluorescence images of the functionalized AAO substrate displaying hydrophobic pore rims and $\mathrm{O}_{2}$ plasma treated pore interiors, after the addition of fluorescently labeled SUVs,

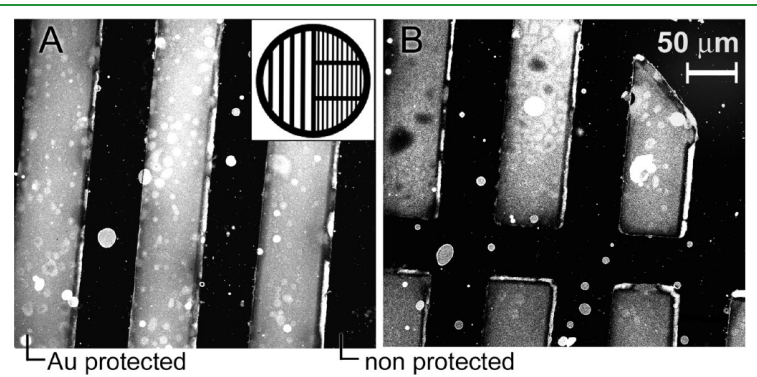

Figure 4. (A, B) Confocal fluorescence fluorescence images of an AAO substrate with pore rims that were selectively silanized with dodecyltrichlorosilane $\left(\mathrm{C}_{12}\right.$-TCS). Au was evaporated through a TEM grid used as a patterning-mask, shown in the inset of (A) to generate the micrometer-sized rectangles. TexasRed-DHPE labeled POPC SUVs adhered to the hydrophobic pore rims functionalized with $\mathrm{C}_{12}$-TCS, whereas they did not bind to the hydrophilic remainder of the unprotected AAO surface (scale bar for both images). are shown. The areas that were Au protected, and thus were functionalized with $\mathrm{C}_{12}$-TCS at the pore rims, show a bright TexasRed fluorescence, whereas the hydroxyl terminated remaining pore rims and pore interiors appear black demonstrating the selective adsorption of lipids on the hydrophobic pore-rim regions.

Pore-Spanning Lipid Membranes. Silane-based surface modifications have the particular advantage that fluorescence near the functionalized surface is not quenched, in contrast to metals where quenching occurs up to $15 \mathrm{~nm}$ away from the surface. ${ }^{48,49}$ On porous substrates with micrometer-sized pores (0.5-2 $\mu \mathrm{m})$, hydrophobic functionalized Au pore rims have been used to induce rupture of giant unilamellar vesicles (GUVs) to form hybrid pore-spanning lipid membranes. However, the fluorescence of the pore rims is quenched by the metal and only the fluorescence in the pore area remains visible. ${ }^{32}$ Using the silane-based functionalization presented in Scheme 1, we prepared a porous AAO substrate with a selective hydrophobic functionalization of the pore rims using $\mathrm{C}_{12}$-TCS (substrate 3, Figure 2A). The POPC GUVs, labeled with TexasRed-DHPE, can rupture on this substrate forming a pore-spanning hybrid lipid membrane as illustrated in Figure 5A. GUVs were first left to incubate with the hydrophobic pore rims, during which time they flatten on the substrate surface. In comparison to SUVs, the large surface area of GUVs allows them to deform and form large flat areas at the interface with the substrate. The flattened area is the pore-spanning membrane. A Z-profile of fluorescence images after the incubation period obtained by CLSM, is shown in Figure $5 \mathrm{~B}$, and illustrates how the vesicles deform on the AAO surface; the fluorescence image in the $X-Y$ focal plane was taken at the AAO top surface and is focused at the flattened GUV bottom. To obtain pore-spanning membrane patches, the adsorbed vesicles were then gently rinsed until they ruptured. The lipid membrane patches obtained were effectively the bottom of the GUVs, which interacted with the hydrophobic pore rims. The fluorescence of these pore-spanning membranes originates solely from the top of the functionalized $3.5 \mu \mathrm{m}$ thick AAO substrate, which indicates that the lipids are localized in the atop focal plane (Figure 5C).

When AAO was not orthogonally functionalized, as for substrate 1 (Figure 2A), where the entire AAO surface was
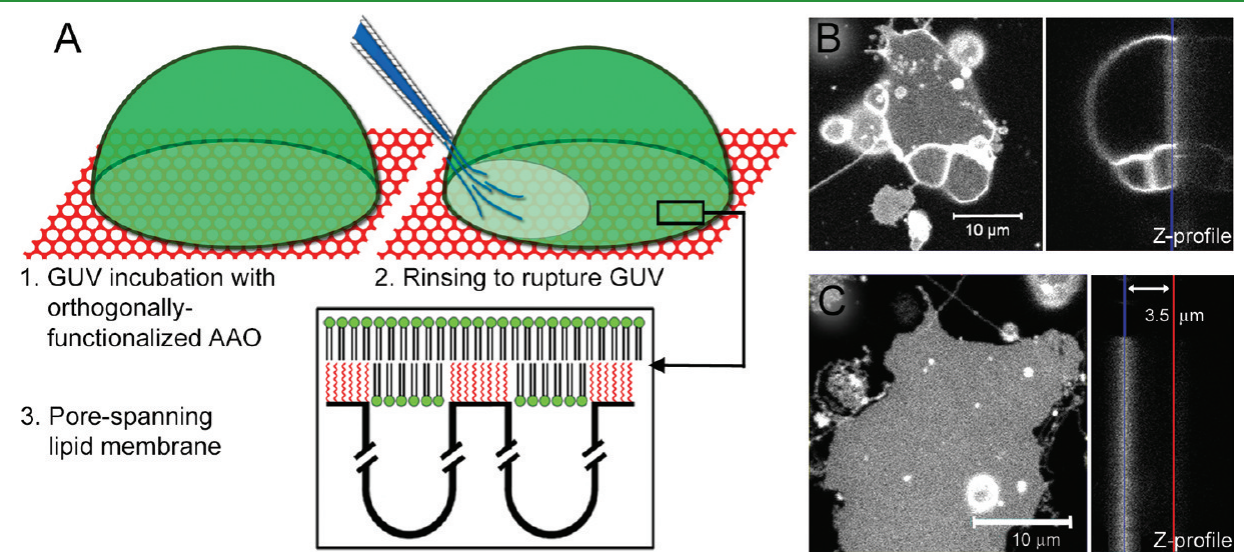

Figure 5. (A) Illustration of the steps leading to the formation of hybrid pore-spanning lipid membranes on orthogonally functionalized AAO with hydrophobic pore rims obtained from silanization with $\mathrm{C}_{12}$-TCS following Scheme 1. (B) Confocal fluorescent image of a giant unilamellar POPC vesicle on an orthogonal functionalized AAO substrate $\left(D_{0}=65 \mathrm{~nm}\right.$ and $\left.l=3.5 \mu \mathrm{m}\right)$ with $C_{12}$-TCS on the pore rims: $X-Y$ plane (left) and Z-profile (right). (C) Confocal fluorescent image of a hybrid lipid membrane patch formed by the rupture of flattened POPC GUVs: the membrane, which is visible in the $X-Y$ focal plane (left) and the Z-profile (right) shows that the fluorescence is localized atop the AAO, not within the AAO. The blue line indicates the top of the $3.5 \mu \mathrm{m}$ thick substrate, whereas the red line indicates the bottom. 


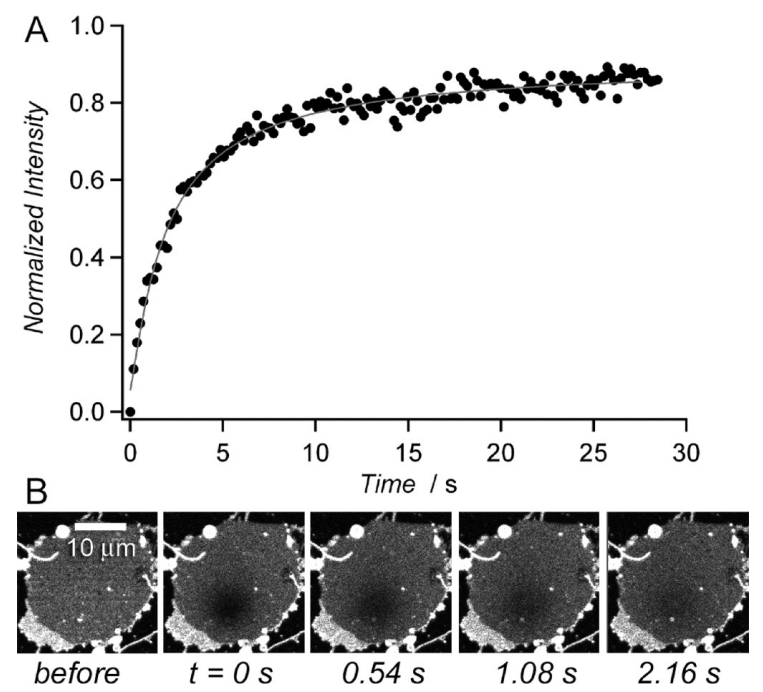

Figure 6. (A) FRAP recovery curve obtained on a pore-spanning hybrid POPC lipid membrane patch. The solid line is the result of the fitting procedure. (B) FRAP experiment carried out on an isolated porespanning lipid membrane patch obtained from GUV rupture. Frames from time series taken at different times during the fluorescence recovery shown in A.

hydrophobic, the volume beneath the measured patch was fluorescent throughout the thickness of the AAO due to lateral lipid diffusion within the membranes lining the pore walls, as observed in the results obtained with SUVs incubated with substrate 1. Using Bodipy-DHPE labeled POPC GUVs, we were able to perform fluorescence recovery after photobleaching (FRAP) experiments on membrane patches.

In Figure 6, a typical FRAP experiment is shown, where the reference was taken near the edge of the patch. Recovery was typically about $80 \%$. During bleaching, fluorescence originating from the lipids in the lower leaflet is lost and does not recover because these lipids are immobile because of the hydrophobic pore rims that confine them (Figure 5A, step 3). The recovery observed is therefore expected to come predominantly from qthe top lipid leaflet, whose fluorescence can fully recover. The diffusion coefficient obtained was $D_{\text {lipids }}=7 \pm 3 \mu \mathrm{m}^{2} \mathrm{~s}^{-1}$. The rather large error is due to the limited size of the membrane patches that only permits bleaching areas with Gauss radii $<10$ $\mu \mathrm{m}$. Despite this error, we can conclude that a planar homogeneous bilayer was formed, which leads to a uniform fluorescence recovery with a diffusion coefficient characteristic for continuous planar lipid membranes. ${ }^{50-53}$ These observations further support the formation of pore-spanning membranes.

\section{CONCLUDING REMARKS}

The challenge in the fabrication of differential porous substrates is to provide a selective pore-rim functionalization, without simultaneous functionalizing the pore-interior surface. Here, we have developed a strategy based on silane chemistry to generate orthogonally functionalized nanoporous anodic aluminum oxide (AAO) substrates, where the pore-rim surface chemistry is distinctly different from the pore-interior surface. The advantage of this strategy is the possibility to carry out a wide range of well-established anhydrous or aqueous solutionphase, as well as gas-phase silanization procedures. Furthermore, the deposition of the protective Au mask and the subsequent $\mathrm{O}_{2}$ plasma removal of the undesired pore-interior functionality are all carried out under vacuum conditions that optimizes the stability of the functionalized surface in comparison to solution treatments. A vacuum environment proves useful for different air/moisture and $\mathrm{pH}$-sensitive surface chemistries. Plasma treatments can also be performed with other gases than $\mathrm{O}_{2}$ and also with reactive monomer species to modify and impart the surfaces of materials with various functionalities. ${ }^{54,55}$ By using silane-based chemistry on AAO substrates, the material remains optically transparent. Owing to this transparency of AAO, it can be used as an optical waveguide, which has allowed us to determine the spatial localization of the adsorption of fluorescently labeled lipids atop and within the pores by confocal laser scanning fluorescence microscopy. In addition, the deposition of nonlabeled molecules was quantified by optical waveguide spectroscopy. We believe that this type of functionalization methodology can also be applied toward other types of porous substrates, where the pore-rim surface is required to be chemically distinct from the surface within the porous channels. The silane-based strategy will pave the way to design multifunctional surfaces with pore-spanning membranes, including membranes built from polymer-multilayers, from polymer brushes, from in situ porerim polymerizations, or from various layer-by-layer assemblies. We have already demonstrated that the formation of hybrid porespanning lipid membranes from GUV rupture is feasible. Such lipid membrane patches on orthogonally functionalized AAO substrates have the potential to be used to study membrane permeability properties by monitoring the transport kinetics across membranes through optical imaging techniques that allow the study of micrometer-sized areas of interest. Furthermore, pore-lining membranes may also be of potential interest for the tailored collection of membrane proteins from solution mixtures.

\section{EXPERIMENTAL SECTION}

Materials. 1-Palmitoyl-2-oleoyl-sn-glycero-3-phosphocholine (POPC) was purchased from Avanti Polar Lipids Inc. (Alabaster, AL, USA). 2-(4,4-difluoro-5-methyl-4-bora-3a,4adiaza-s-indacene-3-dodecanoyl)-1-hexadecanoyl-sn-glycero-3phosphocholine (Bodipy-DHPE) was purchased from Invitrogen (Eugene, OR, USA). TexasRed-DHPE was purchased from Biotinum Inc. (Hayward, CA, USA). Disodium hydrogen phosphate, and oxalic acid dihydrate were purchased from AppliChem (Darmstadt, Germany). Perchloric acid (70\%) and phosphoric acid (85\%) were purchased from Merck (Darmstadt, Germany). Sucrose was purchased from Acros Organics (Geel, Belgium). Ethanol was p.a. grade (VWR, Germany). The water used was ion exchanged and filtered using a Millipore system (Milli-Q System from Millipore, Molsheim, France; specific resistance $R>$ $\left.18 \mathrm{M} \Omega \mathrm{cm}^{-1}, \mathrm{pH} \sim 5.5\right)$. High refractive index LaSFN9 glass substrates $(\varepsilon=3.406, \lambda=632.8 \mathrm{~nm})$ were obtained from Hellma Optik (Halle, Germany). Aluminum foils ( $0.25 \mathrm{~mm}$ thick, purity: 99.999\%) were purchased from Goodfellow (Huntington, UK). The UV-curable optical adhesive (NOA 83H) was purchased from Norland Products (Cranbury, NJ, USA). $\mathrm{CuCl}_{2}$, dodecyltrichlorosilane $\left(\mathrm{C}_{12}\right.$-TCS $)$ and sodium chloride were purchased from Sigma Aldrich (St-Louis, MO, USA). Toluene was p.a. grade (VWR, Germany) and dried over $0.4 \mathrm{~nm}$ molecular sieves from Roth (Karlsruhe, Germany). Indium tin oxide (ITO) slides were from Präzisions Glas \& Optik GmbH (Iserlohn, Germany). 
Scanning Electron Microscopy (SEM). SEM measurements were performed with a LEO Supra-35 SEM from Zeiss with acceleration voltages between 6 and $10 \mathrm{kV} .50 \mathrm{~nm}$ of Au were evaporated onto the samples before measuring.

Au Evaporation and Removal. Au and $\mathrm{Cr}$ were evaporated on a Bal-Tec MCS610 evaporator. For the metal coupling layer in OWS measurements, $2 \mathrm{~nm}$ of $\mathrm{Cr}$, and $25 \mathrm{~nm}$ of Au were evaporated on the AAO barrier layer. When Au was used as a protective mask for orthogonal functionalization, only $10-20 \mathrm{~nm}$ of $\mathrm{Au}$ were evaporated directly onto the $\mathrm{AAO}$ pores at a high deposition rate of $1-2 \mathrm{~nm} \mathrm{~s}^{-1}$. Au was removed with an iodine solution $(50 \mathrm{~g} / \mathrm{L}$ $\mathrm{KI}, 12.5 \mathrm{~g} / \mathrm{L} \mathrm{I}_{2}$ ), filtered through a PTFE $200 \mathrm{~nm}$ filter.

$\mathrm{O}_{2}$ Plasma Treatment. $\mathrm{O}_{2}$ plasma treatments were carried out with a Harrick plasma cleaner (Ithaca, NY, USA). The vacuum chamber was flushed with $\mathrm{O}_{2}$ and evacuated with an oil pump for $2 \mathrm{~min}$. The plasma was turned on and the $\mathrm{O}_{2}$ pressure in the chamber increased to obtain a faint purple plasma. The samples with a protective Au mask were exposed for about $60 \mathrm{~s}$, starting with the initiation of the purple plasma.

Fluorescence Microscopy. CLSM measurements were performed on an upright confocal microscope from Carl Zeiss MicroImaging GmbH (Jena, Germany), model LSM 710 Axio Examiner. A $63 \times$ magnification water immersion objective from Zeiss was used, WPlan-APO-CHROMAT (N.A. = 1.0). The upright epi-fluorescence optical microscope, model Olympus BX-51, was equipped with a filter for Nile Red (UMNG2), both purchased from Olympus Germany $\mathrm{GmbH}$ (Hamburg, Germany). A water immersion objective was used, $40 \times$ magnification from Olympus: LUMPlanF1 40XW (N.A. $=0.8$ ).

Optical Waveguide Spectroscopy (OWS). OWS measurements of the AAO membranes prepared on glass slides were performed on a purpose-built setup. The glass-side was attached to the base of a symmetric LaSFN9 glass prism by optical immersion oil $(\varepsilon=2.89)$. The laser $(\lambda=632.8 \mathrm{~nm})$ was incident through the prism-substrate assembly and reflected off the thin metal coupling layer in between the $\mathrm{AAO}$ and the optical adhesive as the incidence angle $(\theta)$ was varied. At specific $\theta$ 's determined by the thickness and the dielectric constant of AAO $\left(\varepsilon_{\text {AAO }}\right)$, the laser was coupled into the AAO film and these waveguide modes were recorded as sharp minima in a reflectivity $R$ vs $\theta$ scan. Transverse electric (TE) and transverse electric (TM) modes were indexed according to the number of nodes in their electromagnetic field distributions. ${ }^{56} \varepsilon_{\mathrm{AAO}}$ and the thickness of the AAO film, were obtained by fitting ${ }^{56-58}$ the angles of the waveguide mode reflectivity minima using Fresnel simulations carried out with Winspall program. ${ }^{59}$ Tracking the coupling angle of a mode enables real time, in situ monitoring of changes in the dielectric constant of the film, i.e., adsorption kinetics.

Effective Medium Theory Analysis. The $\varepsilon_{\mathrm{AAO}}$ measured by OWS includes contributions from the alumina, the pore-filling medium (e.g., water), and any organic thin layer coating the pore surfaces (i.e., silanes, phospholipids). $\varepsilon_{\mathrm{AAO}}$ has an anisotropic component that is described by the infinite, prolate ellipsoid approximation within the Maxwell-Garnett theory, and well-described elsewhere. $^{60-62}$

$$
\begin{gathered}
\varepsilon_{\text {AAO }}^{\perp}=\varepsilon_{\text {alumina }}+f_{\text {pore }}\left(\varepsilon_{\text {pore }}-\varepsilon_{\text {alumina }}\right) \\
\varepsilon_{\text {AAO }}^{/ /}=\varepsilon_{\text {alumina }} \frac{\varepsilon_{\text {alumina }}+\frac{1}{2}\left(1+f_{\text {pore }}\right)\left(\varepsilon_{\text {pore }}-\varepsilon_{\text {alumina }}\right)}{\varepsilon_{\text {alumina }}+\frac{1}{2}\left(1-f_{\text {pore }}\right)\left(\varepsilon_{\text {pore }}-\varepsilon_{\text {alumina }}\right)}
\end{gathered}
$$

where $\varepsilon_{\mathrm{AAO}}^{\perp}$ and $\varepsilon_{\mathrm{AAO}}^{\prime /}$ are, respectively, the dielectric constant components normal and parallel to the AAO membrane surface, $f_{\text {pore }}$ is the pore volume fraction within the $\mathrm{AAO}, \varepsilon_{\text {alumina }}=$ $2.68^{61}$ is the dielectric constant of bulk anodic alumina at $\lambda=$ $632.8 \mathrm{~nm}$, and $\varepsilon_{\text {pore }}$ is the (effective) dielectric constant within the pores. For a blank AAO film in water, $\varepsilon_{\text {pore }}=\varepsilon_{\text {water }}=1.77$. With the addition of an organic film $\left(\varepsilon_{\text {organic }}=2.2\right)$ on the internal pore surfaces (e.g., silane, lipid monolayer), the volume within the pores is occupied by a combination of the organic material and the pore-filling buffer. Recursively applying eqs 1 and 2 for the organic-filled AAO pores, using a new effective $\varepsilon_{\text {pore }}^{\prime}$ for the pore interior, provides $\varepsilon_{\mathrm{AAO}}$ after molecular adsorption.

AAO Membranes on Glass Supports. AAO anodized from bulk aluminum foils were mounted on LaSFN9 glass slides using an optical adhesive according to a previously reported technique. ${ }^{43}$ Briefly, AAO membrane thin films were fabricated by electrochemical anodization of aluminum foils, which were annealed at $500{ }^{\circ} \mathrm{C}$ overnight. Aluminum foils were polished in a solution of perchloric acid/ethanol $(1: 4 \mathrm{v} / \mathrm{v})$ for $15 \mathrm{~min}, 0^{\circ} \mathrm{C}$ at $20 \mathrm{~V}$. They were then anodized for $2 \mathrm{~h}$ in $0.3 \mathrm{M}$ oxalic acid, $1{ }^{\circ} \mathrm{C}$ at $40 \mathrm{~V}$. The alumina was removed with a $5 \%$ vol. phosphoric acid solution for $2-3 \mathrm{~h}$. As prepared aluminum foils were then anodized a second time for $1.5 \mathrm{~h}$ to obtain the desired thickness of about $3.5 \mu \mathrm{m}$. Aluminum was removed from the anodized foil by immersing it in a $17 \mathrm{~g} / \mathrm{L} \mathrm{CuCl}_{2}$ in $18.5 \mathrm{M} \mathrm{HCl}$ solution until the $\mathrm{AAO}$ became visible and no metal remained. Prior to $\mathrm{Al}$ removal, the $\mathrm{AAO}$ side was isolated from solution by immobilization onto a glass slide and sealed using epoxy adhesive. The metal coupling layer evaporated onto the barrier layer of the AAO membranes. This underlying metal is required for OWS studies and also serves as a positioning tool to locate the transparent alumina using the microscope. Norland $83 \mathrm{H}$ adhesive was diluted in tetrahydrofurane (1:10) and spin-coated on LASFN9 glass slides at $20 \mathrm{rpm}$ for 2-3 s. The Au-coated AAO was glued, barrier side down, onto the glass side and UV-cured for $2 \mathrm{~h}$ with a UV hand lamp, with both lamps on $(\lambda=254+354 \mathrm{~nm}, 4 \mathrm{~W})$ from Herolab (Wiesloch, Germany). The pore diameter $D_{0}$ for all AAO membranes was widened to the desired diameter by etching in 5\% vol. phosphoric acid (diameter increase: $0.75 \mathrm{~nm} \mathrm{~min}^{-1}$ ).

$\mathrm{C}_{12}$-TCS Silanization of AAO. The hydrophobic regions on the AAO substrates were obtained by $\mathrm{O}_{2}$ plasma treatment for $2 \mathrm{~min}$, followed by immersion in dry toluene in a desiccator under vacuum. Dodecyl-trichlorosilane ( $\mathrm{C}_{12}$-TCS), $2 \%$ vol., was added and left under vacuum for $15 \mathrm{~min} .{ }^{47,63}$ The substrates were finally immersed in excess dry toluene, dried under $\mathrm{N}_{2}$, and left overnight under vacuum at $65^{\circ} \mathrm{C}$.

Small Unilamellar Vesicles (SUVs). POPC dissolved in chloroform with $2 \mathrm{~mol} \%$ of TexasRed-DHPE or $0.1 \mathrm{~mol} \%$ of Bodipy-DHPE was transferred to a test tube $(0.2 \mathrm{mg})$, air-dried and then dried under vacuum in an oven at $60{ }^{\circ} \mathrm{C}$ for $3 \mathrm{~h}^{64}$ The phospholipid films were rehydrated by adding $1 \mathrm{~mL}$ of $20 \mathrm{mM}$ phosphate buffer (100 mM NaCl, pH 7; PBS buffer), left to hydrate for $30 \mathrm{~min}$, vortexed and sonicated on ice using a tipsonifier for $3 \times 6$ min periods, at $80 \%$ power (sonifier tip, Bandelin Sonoplus, Berlin, Germany). Vesicle solutions of 0.4 $\mathrm{mg} / \mathrm{mL}$ were prepared for fluorescence experiments and 0.1 $\mathrm{mg} / \mathrm{mL}$ for OWS experiments. Vesicle solutions were used immediately after sonication and sonicated every 15 min during the experiment. For CLSM experiments, TexasRed-DHPE vesicles were left to interact for $2 \mathrm{~h}$ with the functionalized AAO substrates and gently rinsed with excess PBS buffer. For FRAP 
experiments on square regions, Bodipy-DHPE labeled SUVs were used. Z-stacks were taken using $100 \mathrm{~nm}$ thick slices over the $10-15 \mu \mathrm{m}$ range that was scanned.

Giant Unilamellar Vesicles (GUVs). GUVs were prepared by electroformation. ${ }^{65}$ Briefly, $50 \mu \mathrm{L}$ of a $1 \mathrm{mg} / \mathrm{mL}$ POPC lipid solution (1 mol \% TexasRed-DHPE) was left to dry on ITO slides and then spread uniformly on the slide using the length of a sterile needle. The slides were left at $65^{\circ} \mathrm{C}$ under a vacuum for at least $3 \mathrm{~h}$. The chamber was assembled using conductive copper tape at the ITO slide edges and a $1 \mathrm{~mm}$ thick square Teflon spacer between them (volume $\sim 1.7 \mathrm{~mL}$ ) and was filled with 0.3 $\mathrm{M}$ sucrose solution. The cycle was carried out at $12 \mathrm{~Hz}$ with voltage increments every $60 \mathrm{~s}$ for $3 \mathrm{~h}$ : starting at $0.05 \mathrm{~V}$, followed by $0.01 \mathrm{~V}$ steps until $0.2 \mathrm{~V}$, finally $0.1 \mathrm{~V}$ steps until a constant 1.6 $\mathrm{V}$ was applied for the remaining duration. Afterward, a $5 \mathrm{~Hz}$ square wave was applied for $10 \mathrm{~min}$. GUVs were stored at $4{ }^{\circ} \mathrm{C}$ and used for no longer than 2 weeks. Pore-spanning hybrid lipid membranes were formed on $\mathrm{AAO}$ with hydrophobic pore rims, obtained by the orthogonal functionalization of AAO using $\mathrm{C}_{12^{-}}$ TCS. The AAO was rinsed with p.a. ethanol, and then the measuring chamber was rinsed and filled with $1 \mathrm{~mL}$ of PBS buffer; $50 \mu \mathrm{L}$ of GUV solution was slowly added and incubated for $1 \mathrm{~h}$. The heavier sucrose-filled vesicles sink to the substrate. The chamber was then carefully rinsed with an equal volume of PBS buffer and the sample observed under the microscope. Some vesicles rupture by themselves, but the majority require gentle squirting with a $10 \mu \mathrm{L}$ pipet to rupture. FRAP experiments were performed on circular regions of interest using POPC membranes doped with $0.1 \mathrm{~mol} \%$ Bodipy-DHPE.

\section{ASSOCIATED CONTENT}

S Supporting Information. Fluorescence microscopy of the fluorescence being preserved by the Au mask is shown, as well as the fluorescence loss with plasma treatment duration. Fresnel simulations of the angular shifts of waveguide modes for two scenarios are presented: (1) only atop deposition and (2) deposition atop and within the AAO nanopores. A sample measurement of the OWS spectra before and after deposition of a lipid monolayer onto the hydrophobic AAO, substrate 1, together with the raw data of angular shift and the total angular shift for every waveguide mode measured that indicate whether the deposition occurs only atop or uniformly along the entire $\mathrm{AAO}$ pore depth. Also available are SEM images showing that $\mathrm{Au}$ deposition within the AAO pore interiors is minimal, and photos showing the optical transparency of AAO films. This material is available free of charge via the Internet at http://pubs.acs.org.

\section{AUTHOR INFORMATION}

\section{Corresponding Author}

*E-mail: csteine@gwdg.de.

\section{ACKNOWLEDGMENT}

T.D.L. acknowledges the award of a doctoral scholarship from les Fonds Québécois sur la Nature et les Technologies (FQRNT) and additional financial support from the Göttingen Graduate School for Neurosciences and Molecular Biosciences (GGNB). We thank the SFB 803 for financial support.

\section{REFERENCES}

(1) Li, A. P.; Muller, F.; Birner, A.; Nielsch, K.; Gosele, U. J. Appl. Phys. 1998, 84, 6023.

(2) Masuda, H.; Yamada, H.; Satoh, M.; Asoh, H.; Nakao, M.; Tamamura, T. Appl. Phys. Lett. 1997, 71, 2770.

(3) Nielsch, K.; Choi, J.; Schwirn, K.; Wehrspohn, R. B.; Gosele, U. Nano Lett. 2002, 2, 677.

(4) O'Sullivan, J. P.; Wood, G. C. Proc. R. Soc. London, Ser. A 1970, $317,511$.

(5) Bain, C. D.; Troughton, E. B.; Tao, Y. T.; Evall, J.; Whitesides, G. M.; Nuzzo, R. G. J. Am. Chem. Soc. 1989, 111, 321.

(6) Schreiber, F. Prog. Surf. Sci. 2000, 65, 151.

(7) Sellers, H.; Ulman, A.; Shnidman, Y.; Eilers, J. E. J. Am. Chem. Soc. 1993, 115, 9389.

(8) Gombotz, W. R.; Guanghui, W.; Horbett, T. A.; Hoffman, A. S. J. Biomed. Mater. Res. 1991, 25, 1547.

(9) Tran, Y.; Auroy, P. J. Am. Chem. Soc. 2001, 123, 3644.

(10) Brzoska, J. B.; Benazouz, I.; Rondelez, F. Langmuir 1994, 10, 4367.

(11) Fadeev, A. Y.; McCarthy, T. J. Langmuir 2000, 16, 7268.

(12) Nanci, A.; Wuest, J. D.; Peru, L.; Brunet, P.; Sharma, V.; Zalzal, S.; McKee, M. D. J. Biomed. Mater. Res. 1998, 40, 324.

(13) Fadeev, A. Y.; McCarthy, T. J. Langmuir 1999, 15, 3759.

(14) Liston, E. M.; Martinu, L.; Wertheimer, M. R. J. Adhes. Sci. Technol. 1993, 7, 1091.

(15) Medard, N.; Soutif, J. C.; Poncin-Epaillard, F. Surf. Coat. Technol. 2002, 160, 197.

(16) Templeton, M. K.; Weinberg, W. H. J. Am. Chem. Soc. 1985, 107, 97.

(17) Xia, Y. N.; Whitesides, G. M. Ann. Rev. Mater. Sci. 1998, 28, 153.

(18) Park, S. K.; Noh, J. S.; Chin, W. B.; Sung, D. D. Curr. Appl. Phys. 2007, 7, 180.

(19) Wang, Y. D.; Zang, K. Y.; Chua, S. J. J. Appl. Phys. 2006, 100.

(20) Gorton, L. Biosensors and Modern Biospecific Analytical Techniques, 1st ed.; Elsevier: Amsterdam, 2005.

(21) Soriaga, M. P.; Stickney, J.; A. Bottomley, L.; Kim, Y.-G. Thin Films: Preparation, Characterization, Applications, 1st ed.; Springer: New York, 2002.

(22) Wilbur, J. L.; Whitesides, G. M. In Nanotechnology; Timp, G. L., Ed.; Springer-Verlag: New York, 1999, p 331.

(23) Lee, S. B.; Mitchell, D. T.; Trofin, L.; Nevanen, T. K.; Soderlund, H.; Martin, C. R. Science 2002, 296, 2198.

(24) Mitchell, D. T.; Lee, S. B.; Trofin, L.; Li, N. C.; Nevanen, T. K.; Soderlund, H.; Martin, C. R. J. Am. Chem. Soc. 2002, 124, 11864.

(25) Vlassiouk, I.; Krasnoslobodtsev, A.; Smirnov, S.; Germann, M. Langmuir 2004, 20, 9913.

(26) Figeys, D.; Pinto, D. Anal. Chem. 2000, 72, 330A.

(27) Alvarez, S. D.; Li, C. P.; Chiang, C. E.; Schuller, I. K.; Sailor, M. J. Acs Nano 2009, 3, 3301.

(28) Walt, D. R. Acs Nano 2009, 3, 2876.

(29) Fu, J.; Mao, P.; Han, J. Trends Biotechnol. 2008, 26, 311.

(30) Steinle, E. D.; Mitchell, D. T.; Wirtz, M.; Lee, S. B.; Young, V. Y.; Martin, C. R. Anal. Chem. 2002, 74, 2416.

(31) Banerjee, P.; Perez, I.; Henn-Lecordier, L.; Lee, S. B.; Rubloff, G. W. Nat. Nanotechnol. 2009, 4, 292.

(32) Mey, I.; Stephan, M.; Schmitt, E. K.; Muller, M. M.; Ben Amar, M.; Steinem, C.; Janshoff, A. J. Am. Chem. Soc. 2009, 131, 7031.

(33) Schmitt, E. K.; Vrouenraets, M.; Steinem, C. Biophys. J. 2006, 91, 2163.

(34) Hobler, C.; Bakowsky, U.; Keusgen, M. Phys. Status Solidi A 2010, 207, 872.

(35) Popat, K. C.; Mor, G.; Grimes, C. A.; Desai, T. A. Langmuir 2004, 20, 8035.

(36) Velleman, L.; Triani, G.; Evans, P. J.; Shapter, J. G.; Losic, D. Microporous Mesoporous Mater. 2009, 126, 87.

(37) Mutalib Md Jani, A.; Anglin, E. J.; McInnes, S. J. P.; Losic, D.; Shapter, J. G.; Voelcker, N. H. Chem. Commun. 2009, 3062. 
(38) Busby, M.; Kerschbaumer, H.; Calzaferri, G.; De Cola, L. Adv. Mater. 2008, 20, 1614.

(39) Kilian, K. A.; Bocking, T.; Gaus, K.; Gooding, J.J. Angew. Chem., Int. Ed. 2008, 47, 2697.

(40) Sailor, M. J.; Link, J. R. Chem. Commun. 2005, 1375.

(41) Brevnov, D. A.; Barela, M. J.; Brooks, M. J.; Lopez, G. P.; Atanassov, P. B. J. Electrochem. Soc. 2004, 151, B484.

(42) Jee, S. E.; Lee, P. S.; Yoon, B. J.; Jeong, S. H.; Lee, K. H. Chem. Mater. 2005, 17, 4049.

(43) Lazzara, T. D.; Lau, K. H. A.; Knoll, W. J. Nanosci. Nanotechnol. 2010, 10, 4293.

(44) Aronsson, B. O.; Lausmaa, J.; Kasemo, B. J. Biomed. Mater. Res. 1997, 35, 49.

(45) Harrick-Plasma. Applications Involving Plasma Treatments with Harrick Plasma Devices; http://www.harrickplasma.com/applications_ cleaning.php, 2010.

(46) PLASMAtech-literature Ultra-pure Cleaning with Low Pressure Gas Plasma; PLASMAtech, Inc.: Erlanger, KY, 2007.

(47) Fliniaux, O.; Elie-Caille, C.; Pantigny, J.; Bourdillon, C. Electrochem. Commun. 2005, 7, 697.

(48) Liebermann, T.; Knoll, W. Colloids Surf. A-Physicochem. Eng. Asp. 2000, 171, 115.

(49) Vasilev, K.; Knoll, W.; Kreiter, M. J. Chem. Phys. 2004, 120, 3439 .

(50) Dietrich, C.; Bagatolli, L. A.; Volovyk, Z. N.; Thompson, N. L.; Levi, M.; Jacobson, K.; Gratton, E. Biophys. J. 2001, 80, 1417.

(51) Fortig, A.; Jordan, R.; Graf, K.; Schiavon, G.; Purrucker, O.; Tanaka, M. Macromol. Symp. 2004, 210, 329.

(52) Han, X. J.; Pradeep, S. N. D.; Critchley, K.; Sheikh, K.; Bushby, R. J.; Evans, S. D. Chem.-Eur. J. 2007, 13, 7957.

(53) Stottrup, B. L.; Veatch, S. L.; Keller, S. L. Biophys. J. 2004, 86, 2942.

(54) Chu, P. K.; Chen, J. Y.; Wang, L. P.; Huang, N. Mat. Sci. Eng. R 2002, 36, 143.

(55) Ulbricht, M. Polymer 2006, 47, 2217.

(56) Knoll, W. Annu. Rev. Phys. Chem. 1998, 49, 569.

(57) Gitsas, A.; Yameen, B.; Lazzara, T. D.; Steinhart, M.; H., D.; Knoll, W. Nano Lett. 2010, 10, 2173.

(58) Lazzara, T. D.; Lau, K. H. A.; Abou-Kandil, A. I.; Caminade, A. M.; Majoral, J. P.; Knoll, W. Acs Nano 2010, 4, 3909.

(59) Scheller, A. Winspall: Reflectivity Simulation Program Solving Fresnel Equations, 3.01 ed.; Max-Planck Institute for Polymer Research: Mainz, Germany.

(60) Aspnes, D. E. Thin Solid Films 1982, 89, 249.

(61) Lau, K. H. A.; Tan, L. S.; Tamada, K.; Sander, M. S.; Knoll, W. J. Phys. Chem. B 2004, 108, 10812.

(62) Lau, K. H. A.; Duran, H.; Knoll, W. J. Phys. Chem. B 2009, 113,3179 .

(63) Carson, G.; Granick, S. J. Appl. Polym. Sc. 1989, 37, 2767.

(64) Mayer, L. D.; Hope, M. J.; Cullis, P. R. Biochim. Biophys. Acta 1986, 858, 161 .

(65) Bagatolli, L. A.; Parasassi, T.; Gratton, E. Chem. Phys. Lipids 2000, 105, 135. 